\title{
Akt phosphorylation of zyxin mediates its interaction with acinus-S and prevents acinus-triggered chromatin condensation
}

\author{
C-B Chan ${ }^{1}, \mathrm{X} \mathrm{Liu}{ }^{1}, \mathrm{X}$ Tang $^{1}, \mathrm{H} \mathrm{Fu}{ }^{2}$ and $\mathrm{K} \mathrm{Ye} \mathrm{e}^{*, 1}$
}

Zyxin, a focal adhesion molecule, contains LIM domains and shuttles between the cytoplasm and the nucleus. Nuclear zyxin promotes cardiomyocyte survival, which is mediated by nuclear-activated Akt. However, the molecular mechanism of how zyxin antagonizes apoptosis remains elusive. Here, we report that zyxin binds to acinus-S, a nuclear speckle protein inducing apoptotic chromatin condensation after cleavage by caspases, and prevents its apoptotic action, which is regulated by Akt. Akt binds and phosphorylates zyxin on serine 142, leading to its association with acinus. Interestingly, 14-3-3 $\gamma$, but not $\zeta$ isoform selectively, triggers zyxin nuclear translocation, which is Akt phosphorylation dependent. Zyxin is also a substrate of caspases, but Akt phosphorylation is unable to prevent its apoptotic cleavage. Expression of zyxin S142D, a phosphorylation mimetic mutant, diminishes acinus proteolytic cleavage and chromatin condensation; by contrast, wild-type zyxin or unphosphorylated S142A mutant fails. Thus, Akt regulates zyxin/acinus complex formation in the nucleus, contributing to suppression of apoptosis.

Cell Death and Differentiation (2007) 14, 1688-1699; doi:10.1038/sj.cdd.4402179; published online 15 June 2007

Zyxin is a phosphoprotein that accumulates with integrins at cell-substratum attachment sites. It has a proline-rich domain (PRD) at the $\mathrm{N}$-terminus and multiple LIM domains in $\mathrm{C}$ terminal half. The PRD region displays docking sequences for several proteins in actin assembly and organization, and $\mathrm{SH} 3$ domains that are found in a number of proteins in signal transduction pathways. ${ }^{1-7}$ The LIM domain is a double-zincfinger motif that presents in proteins involved in the regulation of cell proliferation and differentiation., ${ }^{8,9}$ Zyxin shuttles between the nucleus and sites of cell adhesion in fibroblasts, and is thus an excellent candidate for relaying information between these two compartments. ${ }^{10}$ Although some of zyxin family proteins, including Trip6 and Ajuba, affect nuclear transcription, ${ }^{11}$ no definite evidence has yet demonstrated that zyxin is implicated in this process.

Acinus, predominantly located in the nucleus, induces apoptotic chromatin condensation after cleavage by caspases. ${ }^{12}$ It is cleaved at both its $\mathrm{N}$ - and $\mathrm{C}$-termini, generating a p17 active form (amino acids (aa) 228-335), which triggers chromatin condensation. Acinus contains a region similar to the RNA recognition motif of Drosophila splicing regulator Sxl, suggesting that it is implicated in RNA metabolism. Indeed, acinus is a component of functional splicesomes. ${ }^{13}$ Acinus is also found in the apoptosis- and splicing-associated protein (ASAP) complex, which is comprised of SAP18, RNPS1, and distinct isoforms of acinus. Addition of the complex to in vitro splicing assays inhibits RNA processing. ${ }^{14}$ Moreover, knockdown of acinus inhibits oligonucleosomal DNA fragmentation during apoptosis. ${ }^{15}$ Acinus is also a component of exon junction complex (EJC), which is deposited on exon-intron junctions during pre-mRNA splicing, and stimulates gene expression at the RNA level. ${ }^{16}$ Recently, we show that acinus is a physiological substrate of nuclear Akt. Akt phosphorylation of acinus on serine 422 and 573 results in its resistance to caspase cleavage and inhibition of acinus-dependent chromatin condensation. Abolishing acinus phosphorylation by Akt accelerates its proteolytic degradation and chromatin condensation. ${ }^{17}$

It has been reported that atrial natriuretic peptide (ANP)stimulated elevation of cGMP levels in cardiomyocytes triggers nuclear accumulation of Akt, which is associated with zyxin. Nuclear-targeted zyxin induces resistance to cell death. Further, zyxin nuclear translocation requires both activation and nuclear localization of Akt, suggesting that these two molecules might mutually regulate each other's residence in the nucleus. ${ }^{18}$ In the current report, we show that zyxin is a physiological substrate of Akt. Akt phosphorylates zyxin on S142 and regulates its association with acinus-S. Nuclear zyxin prevents acinus apoptotic cleavage and abolishes chromatin condensation. We also demonstrate that the interaction with 14-3-3 $\gamma$ might be a potential mechanism for nuclear translocation of zyxin.

\footnotetext{
${ }^{1}$ Department of Pathology and Laboratory Medicine, Emory University School of Medicine, Room 145, Whitehead Building, 615 Michael Street, Atlanta, GA 30322 , USA and ${ }^{2}$ Department of Pharmacology, Emory University School of Medicine, Room 145, Whitehead Building, 615 Michael Street, Atlanta, GA 30322, USA

*Corresponding author: K Ye, Department of Pathology and Laboratory Medicine, Emory University School of Medicine, Room 145, Whitehead Building, 615 Michael Street, Atlanta, GA 30322, USA. Tel: + 1404712 2814; Fax: + 1404712 2979; E-mail: kye@emory.edu

Keywords: Akt; zyxin; 14-3-3; acinus; chromatin condensation

Abbreviations: aa, amino acids; ANP, atrial natriuretic peptide; ASAP, apoptosis- and splicing-associated protein; CA, constitutively active; CTD, C-terminal domain; EGF, epidermal growth factor; EJC, exon junction complex; KD, kinase-dead; MEF, mouse embryonic fibroblast; NES, nuclear export signal; NGF, nerve growth factor; NTD, N-terminal domain; PRD, proline-rich domain; STS, staurosporine

Received 30.1.07; revised 17.4.07; accepted 02.5.07; Edited by P Mehlen; published online 15.6.07
} 


\section{Results}

The C-terminus of acinus binds to LIM domains of zyxin. To look for the binding targets of acinus, we performed a yeast two-hybrid analysis, using the C-terminal domain (CTD) (aa 228-583) of acinus-S as bait. Four of 10 independent positive clones including a variety of overlapping fragments of zyxin protein were isolated, suggesting that zyxin is a major binding partner for acinus in the nucleus. We observed interactions between the CTD of acinus and zyxin CTD (aa 362-572), regardless of which protein is used as bait or prey (Figure 1a). In vitro binding assay revealed that Flag-acinus bound to GST-zyxin-CTD (aa 380-572), but not to zyxin N-terminal domain (NTD) (aa 1-250 or 1-113), consistent with our yeast two-hybrid findings (Figure 1c). Mapping assay reveals that none of the LIM domains alone was able to bind acinus, which required at least two LIM domains. LIM 2 and 3 domains (aa 440-572) possessed stronger binding affinity than LIM 1 and 2 domains. As a control, GST alone did not bind to acinus (Figure 1c). A diagram of different zyxin truncates used in the assay is shown in Figure $1 \mathrm{~b}$.

\section{Growth factors regulate acinus/zyxin complex formation} in the nucleus. Both acinus (aa 228-583) and (aa 340-583) fragments bound to zyxin in the in vivo interaction assay, demonstrating the interactions occurred between acinusCTD and zyxin in intact cells (Figure 2b). Surprisingly, fulllength acinus failed to bind zyxin. The diagram of different acinus truncates is shown in Figure 2a. To examine whether the interaction between acinus and zyxin is regulated by

\begin{tabular}{cccc}
\hline a Library & \#Transformants & \#his + & \#his + and $\beta$-gal + \\
\hline Human Fetal Brain & 1.5 million & 23 & 10 \\
\hline $\begin{array}{ccc}\text { BD fusion protein } \\
\text { (pPC97) }\end{array}$ & AD fusion protein & Histidine prototrophy & $\beta$-gal Filter Assay \\
\hline Acinus (228-583) & Zyxin (326-572) & + & + \\
Zyxin (326-572) & Acinus (228-583) & + & + \\
Acinus (1-228) & Zyxin (326-572) & - & - \\
\hline
\end{tabular}

b

\begin{tabular}{|c|c|c|c|c|}
\hline & & LIM1 & LIM3 & \\
\hline Zyx (1-583) & & & & \\
\hline Zyx (1-250) & bGST & & & $\sim 52 \mathrm{kDa}$ \\
\hline Zyx (1-113) & bGST & & & $\sim 38 \mathrm{kDa}$ \\
\hline Zyx (380-572) & & bGST & $\square$ & $\sim 48 \mathrm{kDa}$ \\
\hline Zyx (380-498) & & bGST & & $\sim 39 \mathrm{kDa}$ \\
\hline Zyx (440-572) & & bGST & & $\sim 41 \mathrm{kDa}$ \\
\hline Zyx (380-439) & & bGST & & $\sim 33 \mathrm{kDa}$ \\
\hline Zyx (440-498) & & bGST & & $\sim 33 \mathrm{kDa}$ \\
\hline Zyx (499-572) & & $\mathrm{bG}$ & & $\sim 34 \mathrm{kDa}$ \\
\hline
\end{tabular}

C

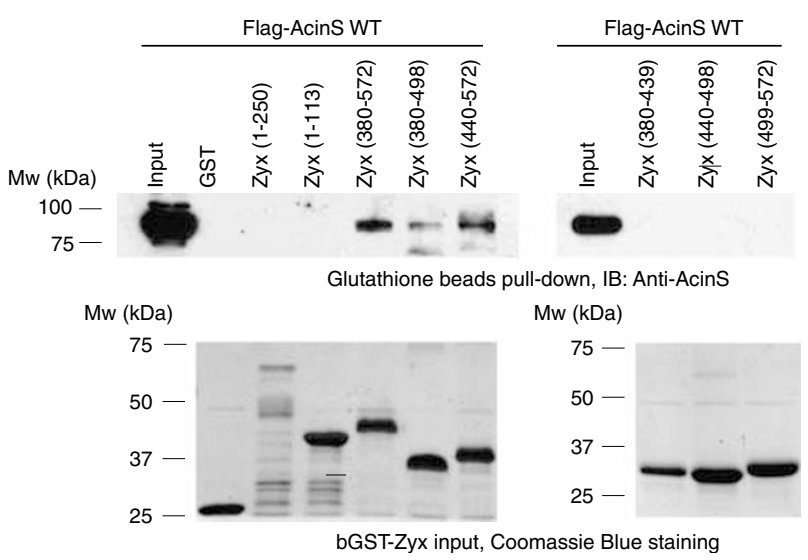

Figure 1 The C-terminus of acinus binds to LIM domain of zyxin. (a) Yeast-two-hybrid screen searching for the binding targets of the CTD of acinus. (b) Diagram of various GST-tagged zyxin deletion mutants. (c) In vitro mapping of the zyxin domains that associate with acinus-S. Purified GST-tagged zyxin proteins were incubated with lysates of HEK293 cells transfected with Flag-tagged acinus-S. The C-terminal end of zyxin (LIM domains), but not the NTD, associates with acinus-S (upper left panel). However, none of the single LIM is able to bind acinus (upper right panel). The GST-fused zyxin fragments used in the in vitro binding were detected by Coomassie blue staining (lower panels) 
a

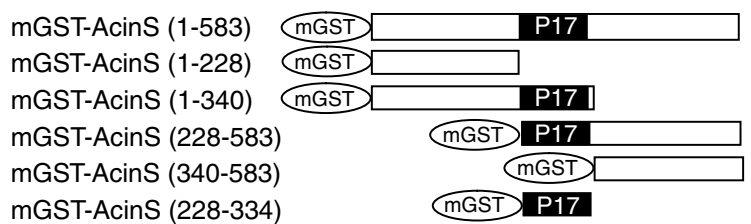

mGST-Acins (228-334)

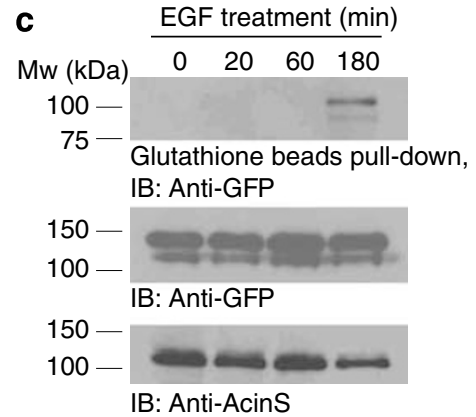

d
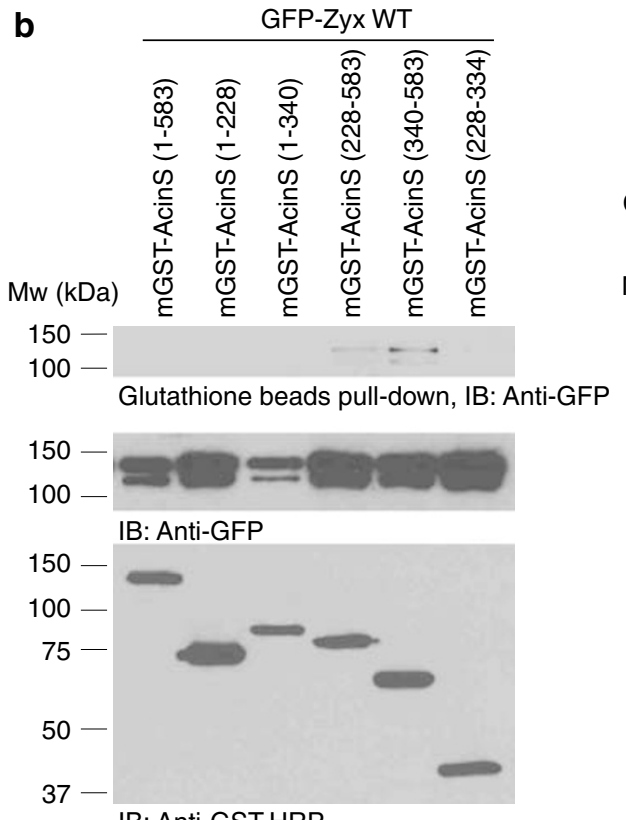

IB: Anti-GST-HRP

Figure 2 Growth factors regulate acinus/zyxin complex formation in the nucleus. (a) A diagram of the different deletion mutants of acinus-S. (b) Mapping of acinus-S domains that associate with zyxin. The C-terminus of acinus-S (aa 340-583) was sufficient to bind full-length zyxin in vivo. Full-length acinus-S, however, did not associate with zyxin (top panel). The expression of GFP-zyxin (middle panel) and GST-acinus-S fragments (bottom panel) was confirmed. (c) EGF treatment triggers the association of acinus-S and zyxin. HEK293 cells cotransfected with GST-acinus-S and GFP-zyxin were stimulated with EGF (50 ng/ml) for different time intervals. Acinus-S was then precipitated using glutathione-Sepharose and bound zyxin was detected using an anti-GFP antibody (top panel). The expression of zyxin (middle panel) and acinus-S (bottom panel) was confirmed. (d) Endogenous association of acinus and zyxin in the nucleus is enhanced upon NGF stimulation. After stimulated by NGF (50 ng/ml) at the indicated times, the cytosolic and nuclear fractions of the PC12 cells were separated and zyxin was immunoprecipitated. The co-precipitated proteins were analyzed using an anti-acinus antibody (first panel). The amount of acinus-S (second panel) and zyxin (third panel) in the cytosolic and nuclear fractions was determined using anti-zyxin and anti-acinus antibodies, respectively. The purity of the cytosolic and nuclear fractions was confirmed by the absence of $\beta$-tubulin in the nuclear fraction and PARP in the cytosolic fraction (fourth and fifth panels)

growth factors, we cotransfected HEK293 cells with GSTacinus and GFP-zyxin and stimulated the transfected cells with epidermal growth factor (EGF). Zyxin associated with acinus after $3 \mathrm{~h}$ of EGF treatment (Figure 2c). Immunofluorescent staining of HEK293 cells, cotransfected with GFP-zyxin and GST-tagged full-length acinus or its CTD, showed that acinus predominantly distributed in the nuclear speckles, whereas zyxin mainly resided in the cytoplasm regardless of growth factor stimulation (data not shown). Colocalization of zyxin and acinus in the nucleus could not be detected in our system, which might be due to the low abundance of nuclear zyxin, as reported. ${ }^{10}$ However, biochemical fractionation of PC12 cells demonstrated that zyxin is present in the nucleus (Figure 2d). To explore whether endogenous acinus binds zyxin, we treated PC12 cells with nerve growth factor (NGF) and prepared nuclear and cytosolic fractions. Acinus strongly bound to zyxin in the nucleus but not cytosol, after 90-180 min of NGF stimulation (Figure 2d). Control IgG displayed no positive signal in either fraction (data not shown). Zyxin mostly occurred in the cytoplasm, whereas acinus primarily resided in the nucleus (Figure 2d). Surprisingly, cytosolic level of acinus decreased upon NGF treatment, whereas nuclear acinus level increased slightly. The purity of nuclear fraction was verified by the absence of $\beta$-tubulin and the presence of its specific marker PARP (Figure 2d). ${ }^{19}$ Therefore, growth factors trigger zyxin and acinus association in the nucleus.

Akt phosphorylates zyxin on serine 142 residue. When exploring the sequence of zyxin, we noticed that aa 138-143 correspond to a motif that is identified as a consensus Akt phosphorylation element present in numerous Akt substrates (Figure 3a). To examine whether zyxin is a substrate of Akt, we tested the ability of recombinant zyxin proteins to be phosphorylated by Akt by in vitro kinase assays, in the presence of $\gamma^{32}$ P-ATP. The NTD fragment (aa 1-250) and positive control GSK $\beta$ were robustly phosphorylated by active Akt (Figure $3 b$ ). Immunoblotting analysis with 
a $\begin{array}{lc}\text { Akt substrate } & \text { RXRXXS } \\ \text { ZYX } & 135 \text { PQP REKVSSIDLEI } 148\end{array}$

b
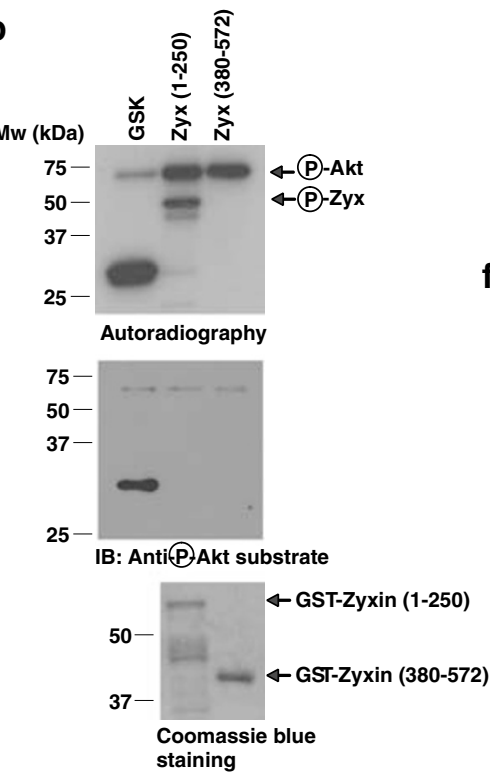

C

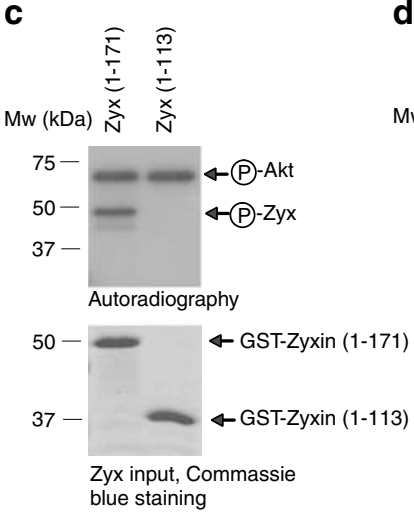

$\mathbf{f}$

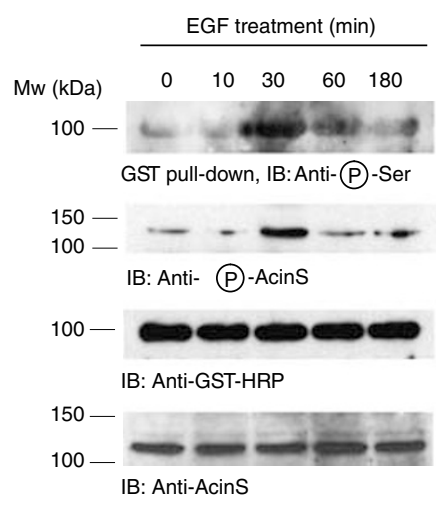

d

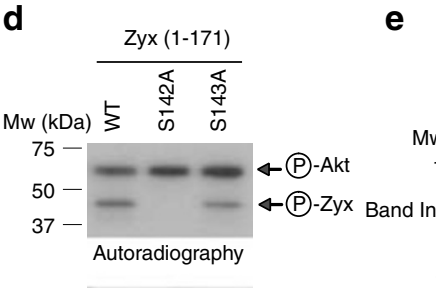

e

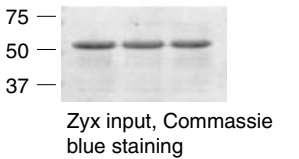

blue staining

g Cytosol \begin{tabular}{llllllllll}
\hline 0 & 10 & 15 & 30 & 180 & 0 & 10 & 15 & 30 & 180
\end{tabular} EGF treatment (min)

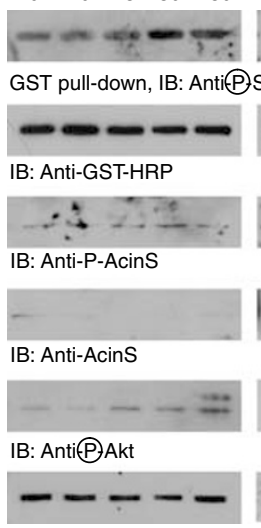
mGST-Zyx mGST-Zyx mGST-Zyx mGST-Zyx
WT S142A

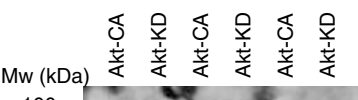
$100-$ 75- 0.010 .061 .190 .660 .170 .57 GST pull-down, IB:Anti®Per

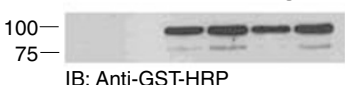
IB: Anti-GST-HRP

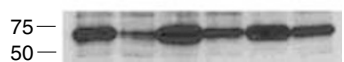

IB: Anti-HA-HRP

Nucleus
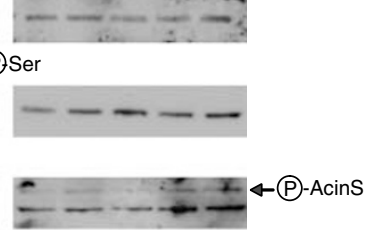

IB: Anti-P-AcinS

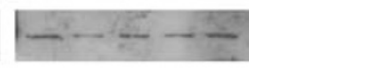

IB: Anti-Akt

Figure 3 Akt phosphorylates zyxin on S142 residue. (a) Identification of the potential phosphorylation sites at the N-terminus of zyxin. This phosphorylation site is colored in red and compared with the consensus Akt phosphorylation site in Akt substrates. (b) Akt phosphorylates the N-terminus of zyxin. GST-tagged N-terminal (aa 1-250) and the C-terminal (aa 380-572) fragments were purified, and used in the in vitro Akt kinase assay (top panel). The phosphorylation, however, could not be recognized by antiphospho-Akt substrate antibody (middle panel). The input of the zyxin fragments was detected by Coomassie blue staining (bottom panel). (c) The Akt phosphorylation site is located between aa 113 and 171 in the N-terminus of zyxin. Two GST-tagged fragments corresponding to 1-171 and 1-113 of zyxin were purified and used in the in vitro Akt assay (upper panel). The fragment (1-171) was phosphorylated by Akt but not the fragment containing aa 1-113. The protein inputs were shown by Coomassie blue staining (lower panel). (d) Identification of Ser ${ }^{142}$ as the Akt phosphorylation site in vitro. S142A but not S143A abolished Akt phosphorylation of the zyxin fragment (upper panel). Equal loading of the proteins in the assay was confirmed by Coomassie blue staining (lower panel). (e) Zyxin is a substrate of Akt in vivo. HEK293 cells were cotransfected with GSTzyxin (wild type or S142A mutant), and either constitutively active Akt (HA-Akt-CA) or KD Akt (HA-Akt-KD). Zyxin was then precipitated using glutathione-Sepharose and its phosphorylation was detected using an anti-phospho-serine antibody. HA-Akt-CA but not HA-Akt-KD phosphorylated wild-type zyxin. No serine phosphorylation was observed in S142A mutant (top panel). Control transfections were also performed in parallel, in which only Akt mutants were transfected. The expression of GST-zyxins (middle panel) and HA-Akt (bottom panel) was confirmed. (f) EGF treatment enhances phosphorylation of zyxin. HEK293 cells were transfected with GST-zyxin and treated with EGF for different time intervals. Zyxin was then precipitated using glutathione-Sepharose and the phosphorylation was detected using an anti-phospho-serine antibody. Zyxin was robustly phosphorylated after EGF stimulation for $30 \mathrm{~min}$. The phosphorylation was gradually decreased until $180 \mathrm{~min}$ (first panel). A similar phosphorylation pattern was observed in endogenous acinus-S, using an anti-phospho-acinus-S antibody (second panel). The expression of GST-zyxin and acinus-S was also confirmed (third and fourth panels). (g) Phosphorylation kinetics of zyxin, acinus and Akt in HEK293 cells. HEK293 cells were transfected with GST-zyxin and treated with EGF for various time intervals, followed by subcellular fractionation. Zyxin was then precipitated using glutathione-Sepharose and the phosphorylation was detected using an anti-phospho-serine antibody. Cytosolic zyxin was highly phosphorylated after EGF stimulation for $30 \mathrm{~min}$, whereas nuclear zyxin phosphorylation was slightly increased after 180 min stimulation (first panel). A similar phosphorylation pattern was observed in endogenous acinus-S in the nucleus, using an anti-phospho-acinus-S antibody (third panel). On the other hand, cytosolic Akt phosphorylation occurred after $15 \mathrm{~min}$ EGF stimulation, whereas nuclear Akt phosphorylation was detected when the cells were stimulated for 30 min (fifth panel). The expression of GST-zyxin, acinus and Akt in both cytosolic and nuclear fractions was also confirmed (second, fourth and sixth panels)

anti-phospho-Akt substrate antibody demonstrates that phosphorylated GSK was recognized by this antibody, whereas phosphorylated zyxin was not (Figure 3b), suggesting that this antibody cannot recognize all Akt substrates. Mapping assay shows that the $\mathrm{N}$-terminal 1-171 but not the 1-113 portion of zyxin could be phosphorylated by Akt, indicating that the 114-171-aminoacid fragment might contain Akt phosphorylation sites (Figure 3c). Mutation with S142A, but not S143A, in zyxin abolished the phosphorylation by active Akt, indicating that $\mathrm{S} 142$ residue can be phosphorylated by Akt in vitro
(Figure 3d). In vivo phosphorylation assay showed that constitutively active (CA, T308DS473D) Akt triggers potent serine phosphorylation in wild-type zyxin but not S142A mutant. In contrast, zyxin phosphorylation was diminished in the presence of kinase-dead (KD, K179A) Akt, suggesting $\mathrm{S} 142$ residue is specifically phosphorylated by Akt in cells (Figure 3e). Moreover, zyxin was potently phosphorylated by EGF stimulation at 30 min (Figure $3 f$ ). Endogenous acinus phosphorylation by Akt displayed the similar temporal pattern (Figure 3f). To further analyze the phosphorylation kinetics of zyxin, acinus, and Akt, subcellular phosphorylation pattern of 
the proteins were analyzed after EGF stimulation. Consistent with our finding in PC12 cells (Figure 2d), zyxin was mainly localized in the cytosol, but significant amount was found in the nucleus (Figure $3 \mathrm{~g}$ ). Cytosolic zyxin was highly phosphorylated after being stimulated by EGF for $30 \mathrm{~min}$, but nuclear zyxin phosphorylation was slightly increased after $180 \mathrm{~min}$ (Figure $3 \mathrm{~g}$ ). On the other hand, phosphorylation of acinus was detected solely in the nucleus after EGF stimulation (Figure $3 \mathrm{~g}$ ). Akt was primarily resident in the cytoplasm, but translocated into the nucleus after $30 \mathrm{~min}$ stimulation (Figure $3 \mathrm{~g}$ ). Phosphorylation of Akt was detectable in the cytosol at $15 \mathrm{~min}$ after EGF treatment, whereas nuclear Akt phosphorylation occurred $30 \mathrm{~min}$ after treatment (Figure $3 \mathrm{~g}$ ). That zyxin and acinus phosphorylation lagged behind Akt activation is probably due to their nuclear translocation, which normally occurs after 10-30 min growth factor treatment. ${ }^{17,20,21}$ Taken together, zyxin is a physiological substrate of Akt in cells.

Phosphorylated and activated Akt associates with zyxin. To assess which portion of zyxin binds to Akt, in vitro binding assays using various recombinant zyxin proteins and HA-Akt wild type or Akt-CA were conducted. Only zyxin-CTD (aa 380-572), consisting of all three LIM domains, strongly bound to Akt-CA, but not wild-type Akt (Figure 4a). Mapping assay demonstrates that LIM 1 domain in the CTD selectively bound to Akt-CA (Figure 4b). To explore the effect of zyxin phosphorylation by Akt on its
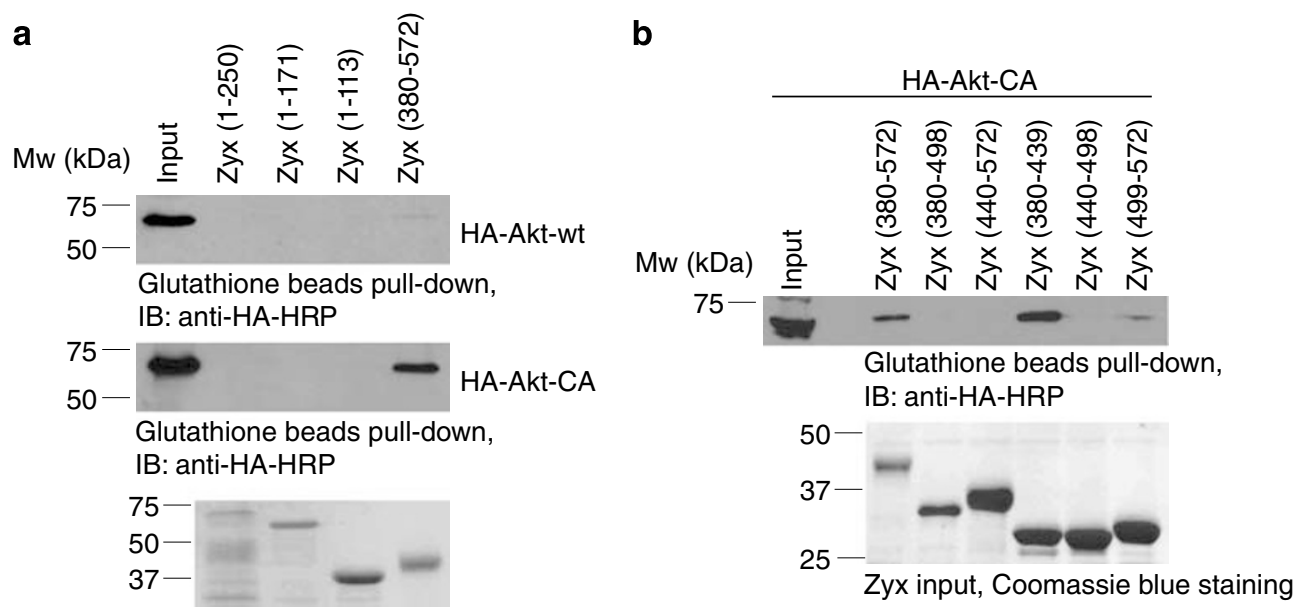

Zyx input, Coomassie blue staining

C
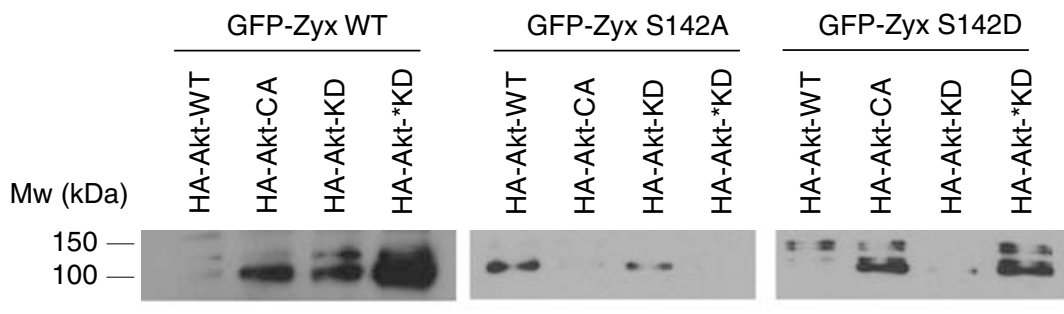

IP: Anti-HA, IB: Anti-GFP
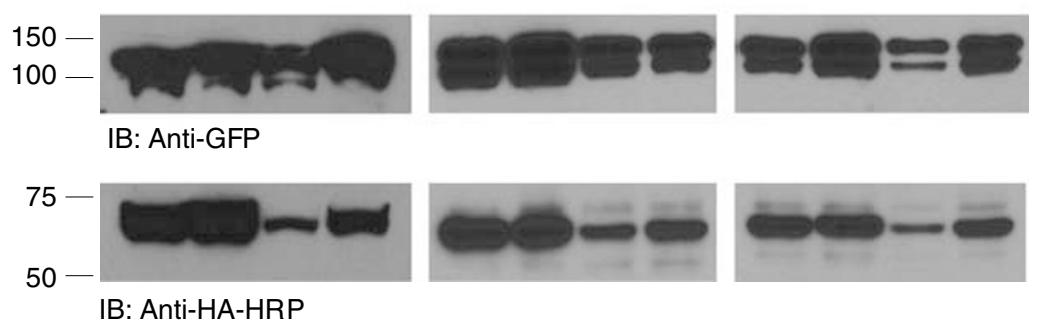

Figure 4 Phosphorylated and activated Akt associates with zyxin. (a) Various GST-tagged zyxin fragments were purified and used in the in vitro binding assay. The LIM domains (aa 380-572) selectively associated with Akt-CA (T308DS473D) (middle panel) but not wild-type Akt (WT) (top panel) from transfected HEK293 cells. The zyxin fragments used in the binding were stained with Coomassie blue (bottom panel). (b) The first LIM domain on zyxin is critical for zyxin/Akt interaction. Zyxin fragments harboring various LIM domains were used in the in vitro binding assay, using cell lysate from HEK293 cells transfected with HA-Akt-CA. HA-Akt-CA interacted with the first and the third LIM domains on zyxin, with a higher affinity to the first LIM domain (upper panel). The zyxin fragments used in the binding were stained with Coomassie blue (lower panel). (c) Phosphorylation of zyxin at Ser142 changes its association with Akt. Various GFP-tagged zyxin constructs (WT, S142A and S142D) were cotransfected with HA-tagged Akt constructs (WT, CA, KD), and phosphorylated KD ('KD, T308DS473DK179A) separately. HA-Akt was precipitated and bound zyxin was detected using an anti-GFP antibody (top panel). Wild-type zyxin and S142D mutant bound strongly with the phosphorylated Akt (CA and $\left.{ }^{*} K D\right)$, whereas S142A mutant preferentially interacted with Akt-KD and wild-type Akt, but with much less affinity. The expression of zyxin and Akt was confirmed (middle and bottom panels) 
association with the upstream kinase, we performed coimmunoprecipitation study. Wild-type zyxin robustly bound to phosphorylated but KD Akt-“KD (T308DS473DK179A), followed by Akt-CA and KD in affinity, fitting with previous observation that zyxin specifically interacts with activated Akt. $^{18}$ Interestingly, zyxin did not bind to wild-type Akt (Figure 4c). Zyxin phosphorylation mimetic mutant, S142D, strongly associated with both Akt-CA and Akt- ${ }^{*} K D$, but neither Akt wild type or KD. Nevertheless, zyxinunphosphorylated mutant, S142A, displayed much weaker affinity to both Akt WT and KD (Figure 4c). Thus, zyxin phosphorylation by Akt dictates its binding activities to Akt.
14-3-3 $\gamma$ interacts with zyxin and stimulates its nuclear translocation. 14-3-3 proteins are phosphoserine/threonine binding proteins that play important roles in many regulatory processes, including intracellular protein targeting. ${ }^{22,23}$ To explore whether zyxin interacts with 14-3-3 proteins, we conducted a co-immunoprecipitation assay. Wild-type zyxin and S142D mutant robustly bound to both $\gamma$ and $\zeta$ isoforms of 14-3-3, whereas S142A mutant failed to bind $\gamma$ isoform, but it still tightly associated with $\zeta$ isoform (Figure $5 \mathrm{a}$ ). To further test the role of Akt phosphorylation on zyxin/14-3-3 $\gamma$ interaction, we treated HEK293 cells with Akt specific inhibitor $(5 \mu \mathrm{M} \mathrm{Akt})$ after cotransfection of GFP-zyxin and a

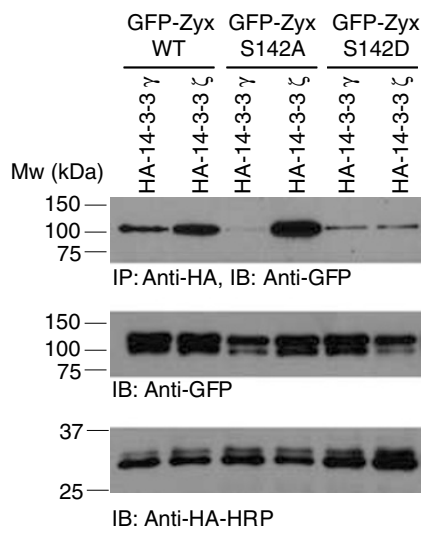

C

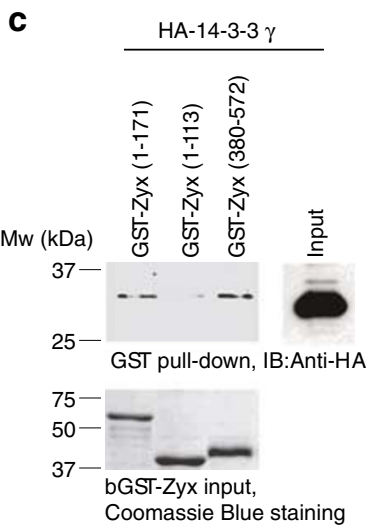

b

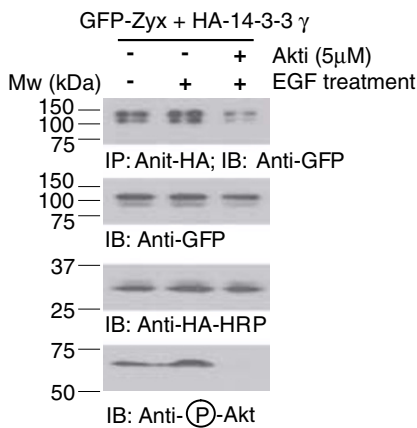

d

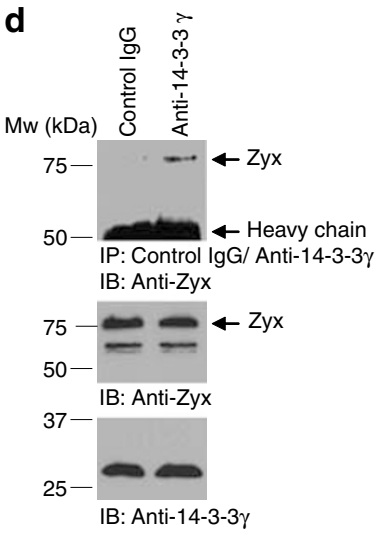

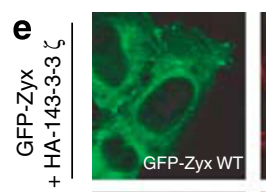
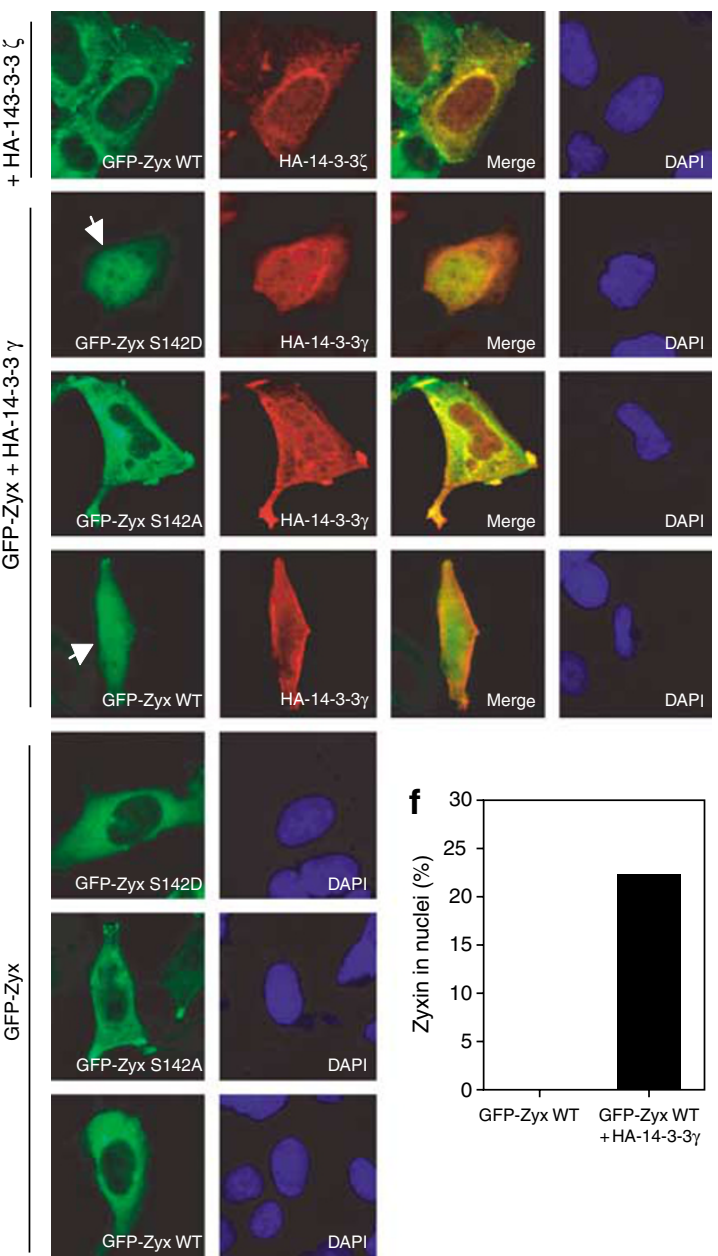

Figure 5 14-3-3 3 interacts with zyxin and stimulates its nuclear translocation. (a) Zyxin binding to 14-3-3 $\gamma$ isoform is Akt phosphorylation dependent. GFP-zyxin wild type, S142A and S142D were cotransfected into HEK293 cells with HA-tagged 14-3-3 $\gamma$ or $\zeta$ isoforms, respectively. 14-3-3 was immunoprecipitated with an anti-HA antibody. 14-3$3 \zeta$ tightly bound to both wild-type and mutant zyxin proteins. 14-3-3 $\gamma$ isoform selectively bound to wild-type and S142D but not S142A (top panel). The expression of GFP-zyxin and HA-14-3-3 was examined and are shown in the middle and bottom panels. (b) Inhibition of Akt diminished the interaction between zyxin and 14-3-3 $\gamma$. HEK293 cells cotransfected with wild-type GFP-zyxin and HA-14-3-3 $\gamma$ were pretreated with $5 \mu$ M Akt-specific inhibitor Akt for $1 \mathrm{~h}$, followed by $50 \mathrm{ng} / \mathrm{ml}$ EGF treatment for $3 \mathrm{~h}$. 14-3-3 $\gamma$ was precipitated by an anti-HA antibody and the bound GFP-zyxin was detected using an anti-GFP-antibody (first panel). The expression of GFP-zyxin (second panel) and HA-14$3-3 \gamma$ (third panel) was confirmed. The action of EGF and Akt was confirmed by examining the phosphorylation of Akt (fourth panel). (c) Both the C-terminal LIM domains and $\mathrm{N}$-terminus interact with 14-3-3. Purified GST-zyxin fragments were incubated with lysates from HEK293 cells, transfected with HA-14-3-3 $\gamma$. Both the C-terminal LIM domains and $\mathrm{N}$-terminal 1-171 fragment of zyxin potently bind to 14-3-3 $\gamma$ isoform (upper panel). The input of the recombinant zyxin proteins was detected by Coomassie blue staining (lower panel). (d) Association of endogenous zyxin and 14-3-3 $\gamma$ in HEK293 cells. Endogenous 14-3-3 $\gamma$ was immunoprecipitated and the bound zyxin was detected using an anti-zyxin antibody. Negative control of IgG was employed in parallel (top panel). Zyxin and 14-3-3y in the cell lysate were also monitored (middle and bottom panels). (e) 14-3$3 \gamma$ but not $\zeta$ isoform selectively provokes zyxin nuclear translocation. GFP-zyxin wild type, S142A and S142D exclusively resided in the cytoplasm (bottom panels); cotransfection with 14-3-3 $\gamma$ triggered wild type and S142D nuclear translocation, whereas S142A still remained in the cytoplasm (middle panels). By contrast, 14-3-3 $\zeta$ failed to elicit any transfected zyxin protein nuclear translocation (top panel) (f) Quantitative analysis of nuclear translocation of transfected zyxin proteins 

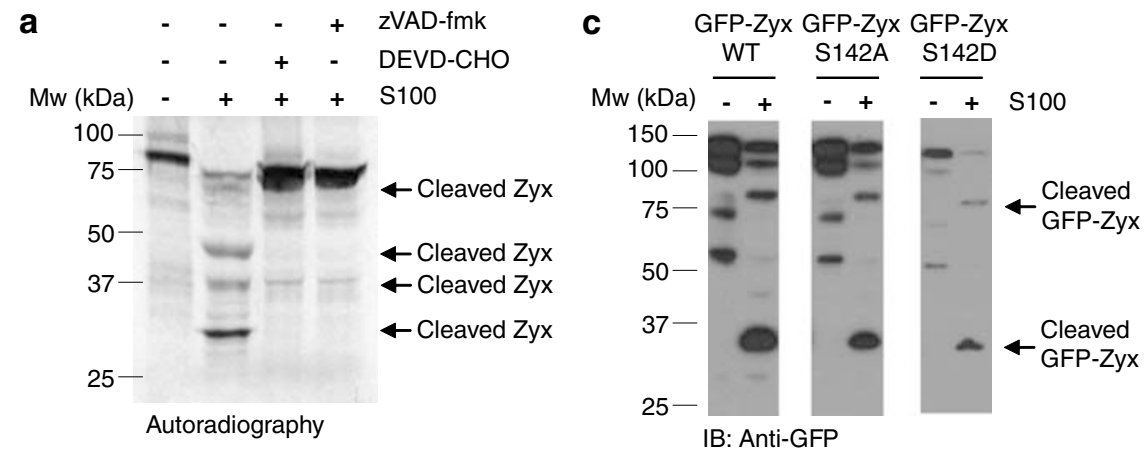

b

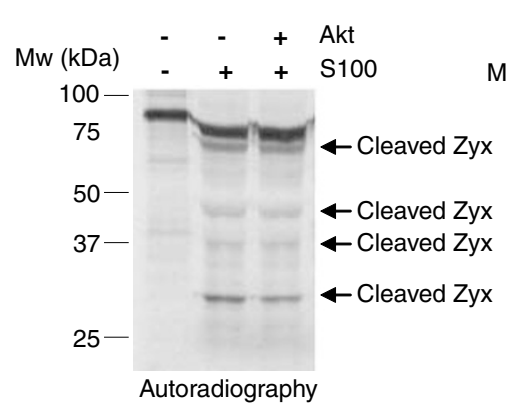

e

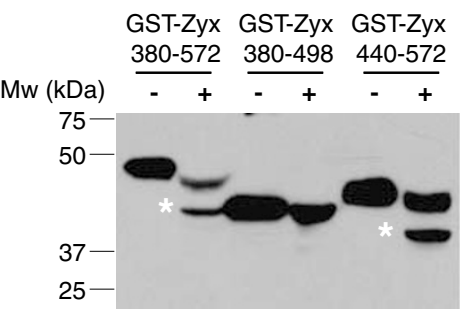

IB: Anti-GST-HRP

f

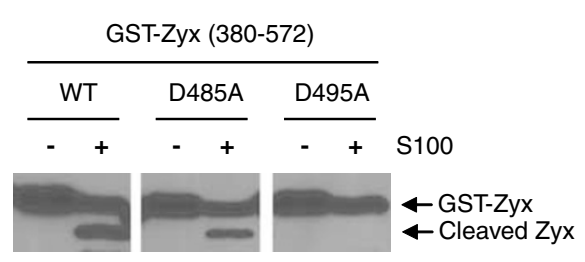

IB: Anti-GST-HRP

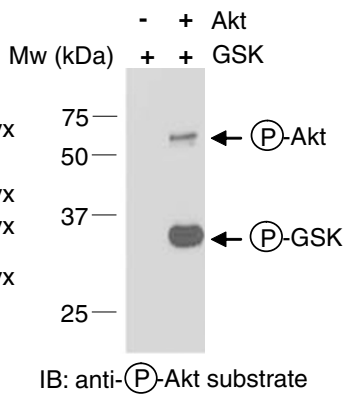

d

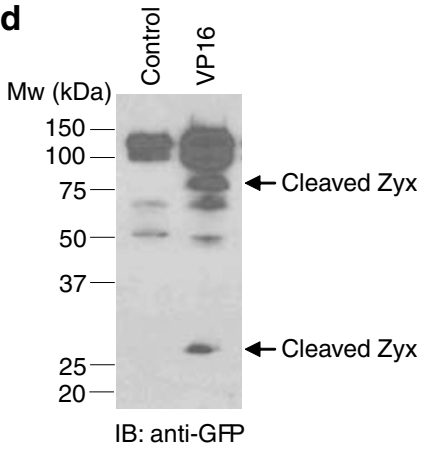

$\mathrm{S} 100$

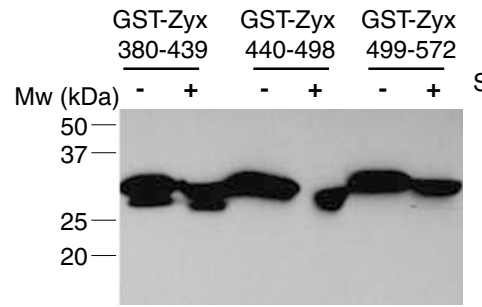

IB: Anti-GST-HRP

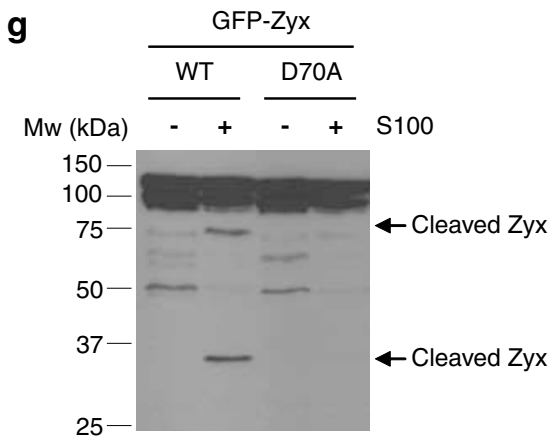

IB: Anti-GFP

Figure 6 Zyxin is a substrate of caspases, but Akt phosphorylation fails to protect its proteolytic degradation. (a) Zyxin is a substrate of caspase in vitro. [ $\left.{ }^{35} \mathrm{~S}\right] \mathrm{methionine-}$ labeled zyxin was prepared by in vitro translation and analyzed in the in vitro caspase cleavage assay. The protein could be cleaved into fragments with sizes about $30,37,48$ and $70 \mathrm{kDa}$. This cleavage was abolished in the presence of general caspases inhibitor zVAD-fmk, (100 $\mu \mathrm{M})$, or a specific inhibitor to caspase 3 (DEVD-CHO, $100 \mu \mathrm{M})$. (b) Phosphorylation of zyxin by Akt does not prevent caspase cleavage. ${ }^{35}$ S $]$ methionine-labeled zyxin was phosphorylated by active Akt, before the in vitro caspase cleavages assay. The Akt phosphorylation, however, did not protect the zyxin from caspase cleavage (left panel). As a positive control, GSK was used as the substrate of Akt in parallel. The phosphorylation of GSK was detected using anti-phospho-Akt substrate antibody (right panel). (c) Mutation of zyxin phosphorylation site does not prevent caspase cleavage in vivo. HEK293 cells were transfected with different GFP-tagged zyxin mutants and cell lysates were incubated with S-100. Cleaved fragments of 30 and $75 \mathrm{kDa}$ were detected. Compared to wild-type zyxin, neither S142A nor S142D mutation prevents the proteolytic cleavage of zyxin. (d) Zyxin could be cleaved in vivo. HEK293 cells transfected with wild-type zyxin were treated with VP16 $(50 \mu \mathrm{M})$ for $24 \mathrm{~h}$. Cleaved fragments of 75 and $30 \mathrm{kDa}$ were detected in VP16-treated but not control cells. (e) Mapping of caspase cleavage site. Various GST-tagged zyxin fragments harboring the LIM domains were used in the in vitro cleavage assay. Only the fragments encompassing the entire LIM regions and the second and third LIM domains could be cleaved by S-100. The cleaved product was mark with an asterisk. (f) Aspartate $e^{495}$ between LIM2 and LIM3 is the caspase cleavage site on zyxin. GST-tagged zyxin fragments with D485A and D495A mutation were used in the in vitro caspase cleavage assay. Mutant D485A but not D495A was cleaved by S-100. (g) Aspartate ${ }^{70}$ at the N-terminus of zyxin is the caspase cleavage site. HEK293 cells were transfected with GFP-tagged wild-type zyxin or D70A mutant, and the cell lysates were then subjected to S-100 cleavage in vitro. Cleavage by S-100 was abolished in D70A mutation 
HA-14-3-3 $\gamma$. While EGF stimulation enhanced the interaction slightly, the binding between zyxin and 14-3-3 $\gamma$ was diminished when Akt was inhibited (Figure 5b). As a control, control IgG in the immunoprecipitation did not reveal any signal (data not shown). These data suggest that Akt phosphorylation regulates the association between zyxin and 14-3-3 $\gamma$, but not the interaction with14-3-3 Truncation assay revealed that the LIM domain and the NTD (aa 1-171) of zyxin potently bound to 14-3-3 $\gamma$. By contrast, the N-terminal 1-113 fragments did not bind to $14-3-3 \gamma$, indicating that the 14-3-3 binding motif on zyxin is located between aa 113-171 (Figure 5c). Coimmunoprecipitation study displayed tight interaction between endogenous zyxin and 14-3-3 $\gamma$ (Figure 5d). To explore whether 14-3-3 affects zyxin subcellular distribution, we performed immunofluorescent staining on HEK293 cells, cotransfected with $\gamma$ or $\zeta$ isoform of 14-3-3 and GFP-zyxin. Surprisingly, only $\gamma$ isoform provoked both wild type and S142D, but not S142A, nuclear translocation, fitting with its binding activity with zyxin. Transfection of zyxin wild type, S142A and S142D alone revealed exclusive cytoplasmic localization (Figure 5e). Quantitative analysis of zyxin nuclear translocation by $14-3-3 \gamma$ is shown in Figure $5 f$.

Zyxin is a substrate of caspases, but Akt phosphorylation fails to protect its proteolytic degradation. Since zyxin carries a few putative caspase cleavage sites, we conducted a cleavage assay using in vitro transcripted and translated zyxin, to investigate the cleavage of zyxin by activated caspases. $\left[{ }^{35}\right.$ S]methionine-labeled zyxin was incubated with activated cell-free apoptotic S-100 solution, ${ }^{24}$ in the presence or absence of caspase-3 or pancaspase inhibitor. Zyxin was cleaved in the active S-100, yielding a few prominent bands of about 30, 37, 48 and $70 \mathrm{kDa}$. The resulting 30 and $48 \mathrm{kDa}$ fragments were abolished in the presence of either caspase-3 or pancaspase inhibitor (Figure 6a). To explore whether Akt phosphorylation affects zyxin cleavage, $\left[{ }^{35}\right.$ S $]$ methioninelabeled zyxin was phosphorylated by active Akt before apoptotic cleavage, in active S-100. Akt phosphorylation of zyxin did not obviously decrease its degradation (Figure 6b). As a control, Akt potently phosphorylated GSK, as manifested by immunoblotting with an anti-phospho-Akt substrate antibody (Figure 6b, right panel). To further test whether Akt phosphorylation of zyxin mediates its proteolytic cleavage in vivo, cell lysate from transfected HEK293 cells was introduced into active apoptosome. Wild-type zyxin was robustly cleaved, yielding 35 and $75 \mathrm{kDa}$ products, indicating that zyxin contains cutting sites on both the $\mathrm{N}$ - and $\mathrm{C}$-termini. Similar apoptotic fragmentation occurred to both S142A and S142D mutants, indicating that Akt phosphorylation might not protect zyxin from proteolytic cleavage (Figure 6c). Treatment of GFP-zyxin-transfected HEK293 cells with VP16 displayed similar cleavage pattern (Figure 6d).

To identify the caspase- 3 cleavage site on zyxin, we performed cleavage assay using different recombinant zyxin proteins. While zyxin fragment containing LIM 2 and 3 domains was robustly cleaved, neither zyxin 380-498 (LIM $1+2$ domains) nor any LIM domain alone was cleaved, suggesting that the cutting site is located between LIM 2 and 3 domains (Figure 6e). Site-directed mutagenesis and in vitro cleavage assays support that D495 is the cutting site at the C-terminus (Figure 6f). Mutation of D70 into D70A completely blocked zyxin cleavage in the $\mathrm{N}$-terminus (Figure $6 \mathrm{~g}$ ). Together, these findings supports that zyxin is a physiological substrate of caspase-3, but Akt phosphorylation on $\mathrm{S} 142$ failed to protect zyxin from caspase-mediated degradation.

Akt phosphorylation of zyxin mediates its interaction with acinus-S, preventing its apoptotic cleavage and chromatin condensation. Co-immunopreciptation was performed to examine the effect of Akt phosphorylation on acinus-zyxin association. Although acinus did not interact with any zyxin proteins in the absence of EGF, EGF stimulation provoked strong association between acinus and zyxin wild type or S142D mutant (Figure 7a). These observations demonstrate that $\mathrm{S} 142$ phosphorylation is necessary but not sufficient for zyxin-acinus interaction. To assess whether Akt-mediated phosphorylation on acinus is sufficient to elicit their interaction, we performed GST-pulldown assay with wild-type and various acinus mutants. While wild-type zyxin did not interact with any of transfected acinus proteins, zyxin S142D evidently bound to acinus (S422DS573D), but it did not bind to wild-type acinus or S422AS573A (Figure 7b). These observations support that growth factor stimulation and Akt-provoked phosphorylation on both zyxin and acinus are indispensable for their association.

To test whether zyxin affects the reported interaction between Akt and acinus, ${ }^{17}$ we investigate the Akt-acinus interaction in the presence of zyxin, after EGF treatment. Robust binding by Akt to acinus was gradually decreased by progressively increased amount of GFP-zyxin. The interaction was still demonstrable, even in the presence of $20 \mu \mathrm{g}$ plasmid of zyxin wild type (Figure 7c). In contrast, transfection of zyxin S142D completely abolished the association between Akt and acinus (Figure 7c), suggesting that S142D possesses much higher affinity to acinus than Akt. Therefore, Aktphosphorylated zyxin competes with Akt for binding to acinus.

To investigate whether zyxin plays any role in mediating acinus apoptotic cleavage, we cotransfected GST-acinus into HEK293 cells with various zyxin constructs, and treated the transfected cells with VP16. Only zyxin S142D mutant strongly prevented acinus proteolytic cleavage (Figure 7d). Moreover, while more than $30 \%$ of GFP control-, wild-type zyxin- and S142A mutant-transfected cells showed condensed chromatin after VP16-staurosporine (STS) treatment, zyxin S142D substantially diminished the chromatin condensation (Figure 7e). The inhibition on chromatin condensation, however, did not affect the final cell fate, as revealed by trypan blue exclusion assay (data not shown). Furthermore, overexpression of nuclear-targeted zyxin substantially decreased VP16-STS-induced chromatin condensation (Figure 7f), fitting with previous report that nuclear zyxin protects cardiomyocyte from apoptosis. ${ }^{18}$ To further investigate whether zyxin is required for repressing chromatin condensation, we eliminated endogenous zyxin with siRNA. Depletion of endogenous zyxin did not affect acinus apoptotic degradation or chromatin condensation in HEK293 cells (data not shown). Transfection of GFP-zyxin S412D significantly decreased 
a

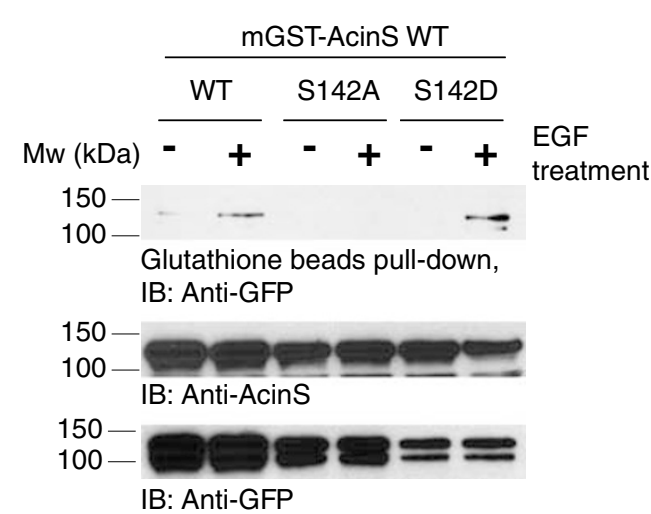

C

\begin{tabular}{|c|c|c|c|c|c|c|c|c|}
\hline \multicolumn{4}{|c|}{ GFP-Zyx WT } & \multicolumn{4}{|c|}{ GFP-Zyx S142D } & \multirow[b]{2}{*}{ HA-Akt-WT } \\
\hline+ & + & + & + & + & + & + & + & \\
\hline+ & + & + & + & + & + & + & + & mGST-AcinS WT \\
\hline 0 & 5 & 10 & 20 & 0 & 5 & 10 & 20 & GFP-Zyx $(\mu \mathrm{g})$ \\
\hline
\end{tabular}

$\begin{array}{llllllllll}\mathrm{Mw}(\mathrm{kDa}) & 0 & 5 & 10 & 20 & 0 & 5 & 10 & 20 & \text { GFP-Zyx }(\mu \mathrm{g})\end{array}$ b

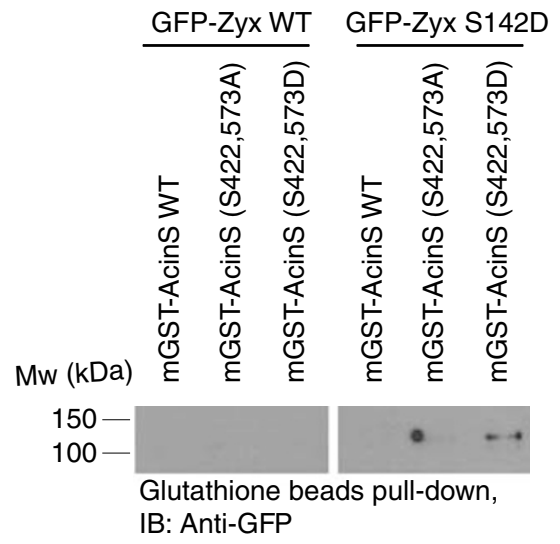

ID: Anti-GST-HRP

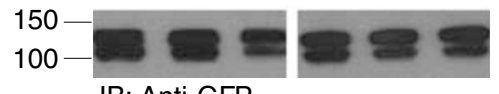

IB: Anti-GFP
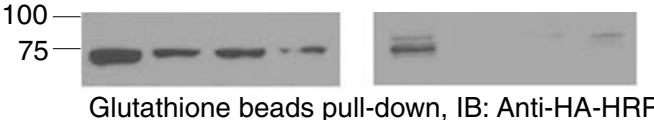

d
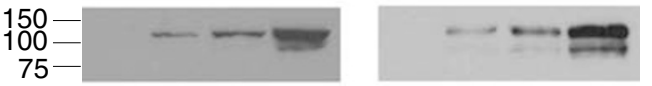

IB: Anti-GFP

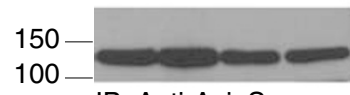

IB: Anti-AcinS
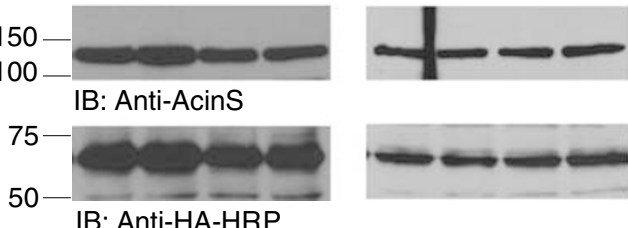

IB: Anti-HA-HRP

e
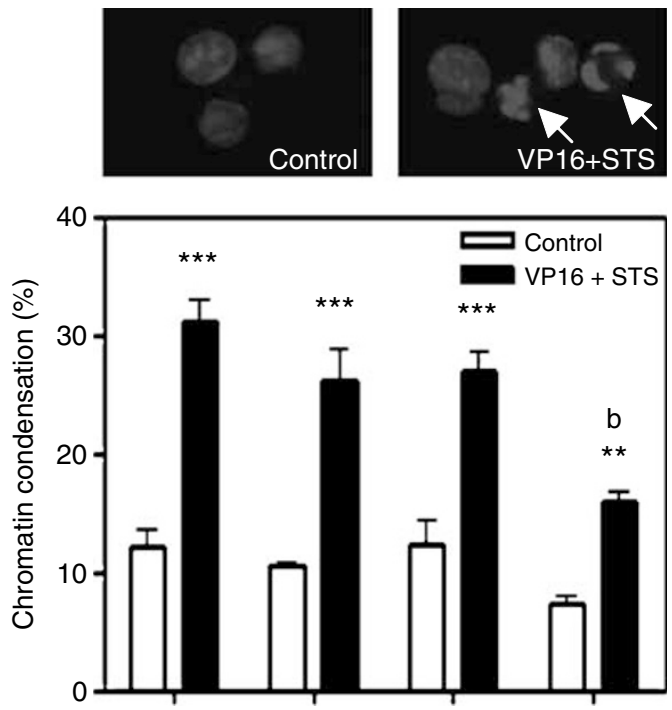

GFP-N1 GFP-Zyx WT GFP-Zyx S142A GFP-Zyx S142D

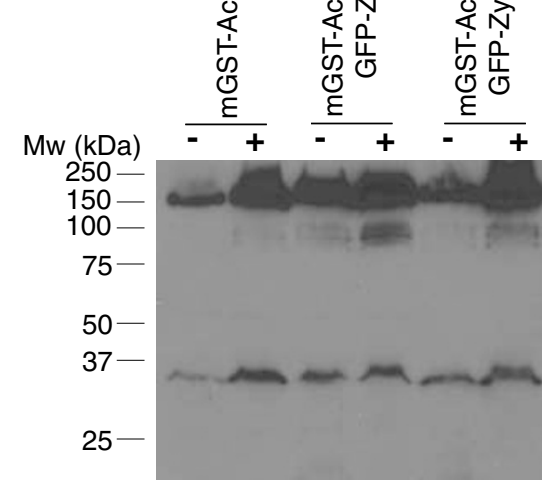

f

IB: Anti-AcinS

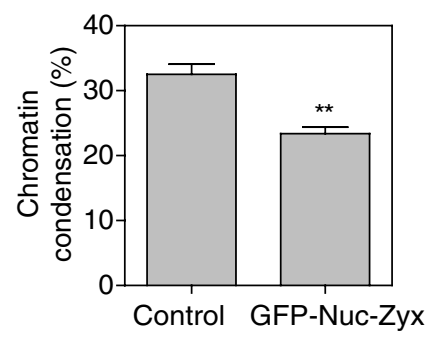

Control GFP-Nuc $-Z y x$

IB: Anti-Zyx

VP16

Acins

$-\mathrm{P} 30$

g

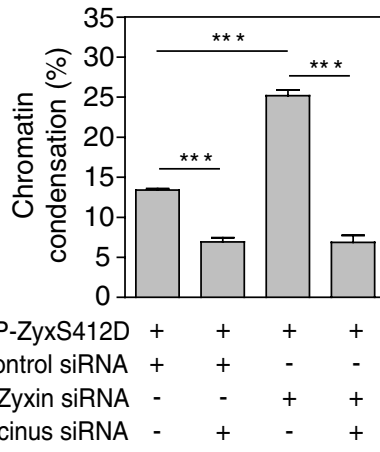


chromatin condensation, whereas depletion of transfected zyxin abolished its inhibitory effect (Figure $7 \mathrm{~g}$ ). In addition, we knocked down both acinus and zyxin in GFP-zyxin S412Dtransfected cells, and found that depletion of acinus alone or both revealed the similar results in chromatin condensation, demonstrating that acinus plays an essential role in provoking chromatin condensation triggered by apoptotic stimuli (Figure 7g). Therefore, Akt-phosphorylated zyxin blocks acinus apoptotic fragmentation and chromatin condensation probably through directly binding to acinus.

\section{Discussion}

In the present study, we have discovered that active Akt tightly interacts with zyxin and phosphorylates it on serine 142 residue. Phosphorylated zyxin associates with acinus-S prevents acinus's apoptotic cleavage and blocks active acinus-triggered chromatin condensation. We also demonstrated a novel interaction between zyxin and 14-3-3 $\gamma$, which might be a potential mechanism for nuclear translocation of zyxin. These data provide a possible mechanistic explanation for the antiapoptotic function of zyxin reported previously. ${ }^{18}$

Zyxin is a LIM protein found prominently at sites of cell adhesion and transiently in cell nuclei. The nuclear accumulation of zyxin occurs within $2.3 \mathrm{~h}$ of nuclear export inhibition initiating by leptomycin $B$ treatment. ${ }^{25}$ This time course is consistent with ANP-elicited zyxin nuclear accumulation in cardiomyocyte, which occurs at about $3.5 \mathrm{~h}$ treatment. ${ }^{18}$ It is also in alignment with our finding that zyxin binds to acinus after $3 \mathrm{~h}$ growth factor stimulation

Previous study reveals that Akt activity is required for zyxin nuclear translocation. ${ }^{18}$ However, how Akt mediates zyxin nuclear import remains elusive. Here, we have investigated the role of Akt phosphorylation on zyxin's subcellular localization. Surprisingly, neither wild-type zyxin nor its S142A or S142D mutant alone resided in the nucleus of HEK293, PC12, NIH3T3 or mouse embryonic fibroblast (MEF), regardless of EGF or NGF stimulation (data not shown). Furthermore, transfection of nuclear Akt in the presence or absence of acinus, or stimulating the transfected cells with cGMP, failed to elicit zyxin nuclear translocation in both HEK293 and PC12 cells (data not shown). Nevertheless, cotransfection with 14-3-3 $\gamma$ selectively provoked wild-type zyxin and S142D, but not S142A nuclear translocation, suggesting that Akt phosphorylation on zyxin is important to its nuclear relocation. This finding is consistent with previous report that nuclear-targeted Akt induces zyxin nuclear translocation, which can be blocked by PI 3-kinase inhibitor LY294002 and wortmannin. ${ }^{18}$ It is noteworthy that nuclear localization of zyxin could only be detected in cells overexpressing 14-3-3 $\gamma$, but not in untransfected cells. This result suggests that only limited amount of 14-3-3 $\gamma$ interacted with zyxin under basal condition, leading to the low abundance of zyxin in the nucleus. Normally, 14-3-3 proteins trap their binding targets in the cytoplasm, but we show herein that they could also elicit the binding partner's nuclear import. We speculate that Akt phosphorylation of zyxin incurs 14-3-3 binding on its phosphorylation motif, in addition to the $\mathrm{C}$ terminal LIM domain, masking its nuclear export signal (NES), thereby abrogating zyxin interaction with the cytoplasmic docking proteins, and triggering its nuclear translocation. Recently, it is showed that importin $\alpha$ binding and the subsequent nuclear import of myopodin are regulated by the serine/threonine phosphorylation-dependent binding of myopodin to $14-3-3 .^{26}$ Therefore, these observations disclose an unappreciated new role for 14-3-3 in promoting the nuclear import of the binding partners.

Nuclear zyxin exhibits potent antiapoptotic activity, ${ }^{18}$ however, the molecular mechanism of how zyxin exerts its protective actions remains obscure. Our data suggest that Akt-phosphorylated zyxin robustly binds to acinus and suppresses its apoptotic cleavage, resulting in inhibition of apoptotic chromatin condensation. The fractionation experiments showed that NGF-triggered interaction selectively occurred in the nucleus, and this interaction was dependent on Akt phosphorylation, because unphosphorylated zyxin

Figure 7 Akt phosphorylation of zyxin mediates its interaction with acinus, preventing its apoptotic cleavage and chromatin condensation. (a) Mutation of the Akt phosphorylation site on zyxin abolished the zyxin/acinus interaction triggered by EGF. HEK293 cells were transfected with GFP-tagged wild type zyxin, S142A or S142D mutants, together with GST-tagged acinus-S. The acinus-S proteins were precipitated and the bound zyxin was detected using an anti-GFP antibody. Enhanced interactions were detected using wild-type zyxin and its S142D mutant. This interaction, however, was abolished when using S142A mutant (top panel). The expression of GST-acinus-S (middle panel) and GFP-zyxin (lower panel) was confirmed.(b) Interaction of acinus-S and zyxin depends on the phosphorylation status. Interaction of zyxin and acinus could only be observed when S142D mutant of zyxin and Akt phosphorylation mimetic S422,573D mutant of acinus-S were cotransfected (top panel). The expression of GSTacinus-S (middle panel) and GFP-zyxin (bottom panel) was confirmed. (c) Akt-phosphorylated zyxin prevents Akt from interacting with acinus-S. HEK293 cells were cotransfected with HA-tagged wild-type Akt, GST-acinus-S and various amount of GFP-tagged wild-type or S142D zyxin. After EGF treatment for $3 \mathrm{~h}$, the acinus-S proteins were precipitated and the bound Akt was detected using an anti-HA-HRP antibody. The binding between Akt and acinus-S was diminished with increased amount of zyxin. This displacement of Akt from acinus-S was more obvious when S142D mutant was used in the cotransfection (first panel). The expression of GFP-zyxin (second panel), GSTacinus-S (third panel) and HA-Akt (fourth panel) was confirmed. (d) Akt-phosphorylated zyxin prevents apoptotic cleavage of acinus-S. HEK293 cells were first cotransfected with GST-acinus-S and wild-type GFP-zyxin, S142A or S142D mutant, followed by treatment with VP16 (50 $\mu \mathrm{M})$ for $24 \mathrm{~h}$. Cleavage of acinus-S was detected. S142D mutant selectively prevented acinus-S cleavage. (e) Apoptotic chromatin condensation was diminished in cells overexpressing GFP-zyxin S142D mutant. HEK293 cells were first transfected with GFP-N1 control vector, GFP-tagged wild-type zyxin, S142A or S142D mutant and treated with DMSO (control) or $100 \mu \mathrm{M}$ VP16 for $2 \mathrm{~h}$, followed by $1 \mu \mathrm{M}$ STS for $9 \mathrm{~h}$. The percentage of nuclei with condensed chromatin (upper panel, indicated by white arrow) was then quantitated and expressed as mean $\pm S$.E.M ( $n=3$ ) from at least 500 nuclei. ( ${ }^{\star \star} P<0.01,{ }^{\star \star \star} P<0.001$ versus control; (b): $P<0.01$ versus GFP-N1, two-way ANOVA). (f) Nuclear-targeted zyxin diminished VP16-STS induced chromatin condensation. HEK293 cells were infected with adenovirus with nuclear-targeted zyxin or control adenovirus for $24 \mathrm{~h}$. The cells were treated with $100 \mu \mathrm{M}$ VP16 for $2 \mathrm{~h}$, followed by $1 \mu \mathrm{M}$ STS for $9 \mathrm{~h}$. The number of cells showing condensed chromatin was significantly inhibited $(P<0.01, n=3)$, from about $33 \%$ to about $23 \%$ in cells with nuclearlocalized zyxin (left panels). The expression of nuclear-targeted zyxin in HEK293 cells in the chromatin condensation assay was verified by immunoblotting analysis (right panels). (g) Knocking down of zyxin abolished the protective effect of GFP-ZyxS142D from VP16-STS-induced chromatin condensation. HEK293 cells were transfected with GFP-ZyxS142D together with siRNA specifically against either zyxin or acinus. Forty-eight hours after transfection, the cells were stimulated with $100 \mu \mathrm{m}$ VP16 for $2 \mathrm{~h}$, followed by $1 \mu \mathrm{M}$ STS for $9 \mathrm{~h}$. The number of cells showing condensed chromatin was significantly increased $\left({ }^{* * *} P<0.001, n=3\right)$, from about $13-25 \%$, in cells with zyxin depletion. Chromatin condensation was significantly $\left.{ }^{* * *} P<0.001, n=3\right)$ diminished when acinus was depleted alone or together with zyxin 
S142A mutant failed to associate with acinus-S, whereas S142D tightly bound to acinus (S422DS573D). Interestingly, Akt-phosphorylated zyxin prevents acinus apoptotic degradation, but unlike acinus, Akt phosphorylation of zyxin is unable to block its proteolytic degradation. The consequences of caspase cleavage on the physiological roles of zyxin remain unclear at the present stage, but we speculate that the cleavage on the $\mathrm{N}$-terminus might cause dissociation of zyxin from the cytoplasmic binding partners, including $\alpha$-actinin, as the N-terminal 21-42 portion of zyxin is the binding site with $\alpha$-actinin. We have reported before that PIKE-A also interacts with Akt, which enhances its kinase activity. ${ }^{27}$ Whether zyxin possesses similar property in mediating, Akt activity awaits further studies. Also, it would be interesting to see if there is any physiological interaction between zyxin and PIKE-A.

Our previous studies demonstrate that Akt phosphorylates acinus on both $\mathrm{S} 422$ and 573 residues, leading to its resistance to caspase cleavage in the nucleus and the inhibition of acinus-dependent chromatin condensation. ${ }^{17}$ Since both zyxin and acinus are Akt kinase substrates, the interaction between zyxin and acinus in the nucleus should be regulated by Akt phosphorylation. As expected, zyxin S142D, but not wild-type zyxin or $\mathrm{S142A}$, displays robust binding affinity to acinus S422, S573D. Therefore, Akt might provide a dual protection on acinus by inducing a direct binding between zyxin and acinus and blocking its proteolytic degradation, in addition to direct phosphorylation on acinus.

Taken together, our data suggested a potential mechanism for the antiapoptotic role of zyxin through protecting acinus-S from apoptotic cleavage. Upon growth factors stimulation, Akt is activated and subsequently transported into the nucleus. ${ }^{21}$ Because zyxin is continuously shuttled between cytoplasm and nucleus (possible through the interaction with 14-3-3 $\gamma$ ), the nuclear-resident zyxin is phosphorylated by Akt, which enhances its binding to acinus-S. This interaction prevents acinus-S from apoptotic cleavage and diminishes chromatin condensation.

\section{Materials and Methods}

PC12 cells were maintained in medium A (DMEM with $10 \%$ fetal bovine serum (FBS), $5 \%$ horse serum and $100 \mathrm{U}$ penicillin-streptomycin) at $37^{\circ} \mathrm{C}$ in a $5 \% \mathrm{CO}_{2}$ atmosphere, in a humidified incubator. All PC12 cells employed in the experiments are in naive but not differentiated form. NGF was from Roche. Anti- $\beta$-tubulin antibodies were from Santa Cruz Biotechnology Inc. (Santa Cruz, CA, USA). AntiMyc, acinus, phospho-Akt-473 and Akt antibodies were from Cell Signaling. Active Akt protein was from Upstate Biotechnology Inc. Human fetal brain library was from Clontech. Chemicals not mentioned above were from Sigma.

Yeast two-hybrid screen. Two-hybrid screening was conducted using Y190 yeast strain containing the HIS3 and $\beta$-galactosidase ( $\beta$-gal) reporter genes and the pAS2-1 and pACT2 expression vectors. The experiments were executed exactly as described. $^{28}$

Cytoplasmic and nuclear fractionation. HEK293 cells and PC12 cells were collected and wash once with ice-cold $1 \times$ PBS. The cell pellet was resuspended in CER I buffer. The cytoplasmic and nuclear fractions were prepared as described in the manufacturer's protocol (PIERCE, Rockford, IL, USA, NE-PER, nuclear and cytoplasmic extraction reagent).

Co-immunoprecipitation and in vitro binding assays. A $10-\mathrm{cm}$ plate containing HEK293 cells or PC12 cells was washed once in PBS, cells were lysed in $1 \mathrm{ml}$ lysis buffer (50 mM Tris, pH 7.4, $40 \mathrm{~mm} \mathrm{NaCl}, 1 \mathrm{~mm}$ EDTA, 0.5\% Triton X-100,
$1.5 \mathrm{mM} \mathrm{Na}_{3} \mathrm{VO}_{4}, 50 \mathrm{~mm} \mathrm{NaF}, 10 \mathrm{~mm}$ sodium pyrophosphate, $10 \mathrm{~mm}$ sodium $\beta$ glycerophosphate, $1 \mathrm{~mm}$ phenylmethylsulfonyl fluoride (PMSF), $5 \mathrm{mg} / \mathrm{ml}$ aprotinin, $1 \mathrm{mg} / \mathrm{ml}$ leupeptin, $1 \mathrm{mg} / \mathrm{ml}$ pepstatin A), and centrifuged for $10 \mathrm{~min}$ at $14000 \times \mathrm{g}$ at $4{ }^{\circ} \mathrm{C}$. The supernatant was transferred to a fresh tube. Experimental procedures for co-immunoprecipitation and in vitro binding assays are as described. ${ }^{28}$ After SDSPAGE, the samples were transferred to a nitrocellulose membrane. Western blotting analysis was performed with a variety of antibodies.

Cytochemical staining of apoptotic cells. Morphological changes in the nuclear chromatin of cells undergoing apoptosis were detected by staining with 4,6diamidino-2-phenylindole (DAPI), as described. ${ }^{29}$ In total, about 500 nuclei were counted in different fields.

Immunofluorescence of zyxin and 14-3-3. HEK293 cells were cotransfected with HA-14-3-3 and GFP-zyxin. Cells were fixed with cold $\left(-20^{\circ} \mathrm{C}\right)$ methanol for $5 \mathrm{~min}$ and then rehydrated by PBS for $1 \mathrm{~min}$. Nonspecific sites were blocked by incubating with $200 \mu \mathrm{l}$ of $1 \% \mathrm{BSA}$ in PBS at $37^{\circ} \mathrm{C}$ for $15 \mathrm{~min}$. A mouse monoclonal antibody against $\mathrm{HA}$ was diluted at $1: 200$ in PBS containing $1 \% \mathrm{BSA}$ and incubated on the coverslips at $37^{\circ} \mathrm{C}$ for $1 \mathrm{~h}$. Cells were then washed with $1 \%$ BSA/PBS for 10 min at room temperature, before incubating with a $1: 200$ dilution of Texas Red-labeled goat anti-mouse IgG antibody at room temperature for $45 \mathrm{~min}$, and then the coverslips were rinsed with a $1 \%$ BSA/PBS solution for $10 \mathrm{~min}$. The cells were then stained with DAPI for another $10 \mathrm{~min}$ at room temperature. The coverslips containing the cells were then mounted with AquaMount (Lerner Laboratories, New Haven, CT, USA) containing $0.01 \%$ 1,4diazobicyclo(2,2,2)octane. Cells were examined under a fluorescence microscope.

In vitro apoptotic cleavage in active $\mathbf{S}-\mathbf{1 0 0}$. Zyxin was subcloned in TNT vector (Promega, Madison, WI, USA), and in vitro transcripted and translated in rabbit reticulocyte in the presence of $\left.{ }^{35} \mathrm{~S}\right] \mathrm{methionine}$. The radiolabeled proteins were incubated with active S-100, prepared from HEK293 cytosolic fraction at $30^{\circ} \mathrm{C}$ for $2 \mathrm{~h}$. The reaction mixture was analyzed by SDS-PAGE. After the gel was dried, it was exposed to a film at $-80^{\circ} \mathrm{C}$ overnight. GFP-tagged zyxin was expressed in HEK293 cells and cleaved in active S-100. The proteolytic products were verified by immunoblotting analysis with an anti-GFP antibody.

Zyxin RNAi knockdown. HEK293 cells were transfected with GFP-zyxin S142D, and the expression of zyxin was knocked down by transfecting cells with $100 \mathrm{nM}$ anti-zyxin siRNA (Dharmacon Research Inc., Chicago, IL, USA) or antiacinus siRNA (Qiagen, Valencia, CA, USA). Control experiment was performed in parallel by using $100 \mathrm{nM}$ non-targeting siCONTROL in the transfection.

Statistical analysis. Results were expressed as mean \pm S.E.M., and were considered significant when $P<0.05$. Statistic analyses of the data were performed using either $t$-test, one-way ANOVA or two-way ANOVA, followed by Tukey's multiple comparison test or Bonferroni post-tests, using the computer program GraghPad Prism (GraphPad Software, USA).

Acknowledgements. This work was supported by grants from National Institute of Health (RO1, NS045627, CA117872) to K Ye. We are thankful to Dr Mark Sussman for NLS-Akt and GFP-NLS-zyxin adenovirus and Dr Jürgen Wehland for the GFP-zyxin construct.

1. Ahern-Djamali SM, Comer AR, Bachmann C, Kastenmeier AS, Reddy SK, Beckerle MC et al. Mutations in Drosophila enabled and rescue by human vasodilator-stimulated phosphoprotein (VASP) indicate important functional roles for Ena/VASP homology domain 1 (EVH1) and EVH2 domains. Mol Biol Cell 1998; 9: 2157-2171.

2. Crawford AW, Michelsen JW, Beckerle MC. An interaction between zyxin and alphaactinin. J Cell Biol 1992; 116: 1381-1393.

3. Drees BE, Andrews KM, Beckerle MC. Molecular dissection of zyxin function reveals its involvement in cell motility. J Cell Biol 1999; 147: 1549-1560.

4. Gertler FB, Niebuhr K, Reinhard M, Wehland J, Soriano P. Mena, a relative of VASP and Drosophila Enabled, is implicated in the control of microfilament dynamics. Cell 1996; 87 227-239.

5. Niebuhr K, Ebel F, Frank R, Reinhard M, Domann E, Carl UD et al. A novel proline-rich motif present in ActA of Listeria monocytogenes and cytoskeletal proteins is the ligand for the EVH1 domain, a protein module present in the Ena/VASP family. EMBO J 1997; 16 5433-5444. 
6. Reinhard M, Zumbrunn J, Jaquemar D, Kuhn M, Walter U, Trueb B. An alpha-actinin binding site of zyxin is essential for subcellular zyxin localization and alpha-actinin recruitment. J Biol Chem 1999; 274: 13410-13418.

7. Hobert $\mathrm{O}$, Schilling JW, Beckerle MC, Ullrich A, Jallal B. SH3 domain-dependent interaction of the proto-oncogene product Vav with the focal contact protein zyxin. Oncogene 1996; 12: 1577-1581.

8. Gill GN. The enigma of LIM domains. Structure 1995; 3: 1285-1289.

9. Sanchez-Garcia I, Rabbitts TH. The LIM domain: a new structural motif found in zincfinger-like proteins. Trends Genet 1994; 10: 315-320.

10. Nix DA, Beckerle MC. Nuclear-cytoplasmic shuttling of the focal contact protein, zyxin: a potential mechanism for communication between sites of cell adhesion and the nucleus. J Cell Biol 1997; 138: 1139-1147.

11. Missero C, Pirro MT, Simeone S, Pischetola M, Di Lauro R. The DNA glycosylase T:G mismatch-specific thymine DNA glycosylase represses thyroid transcription factor-1. activated transcription. J Biol Chem 2001; 276: 33569-33575.

12. Sahara S, Aoto M, Eguchi Y, Imamoto N, Yoneda Y, Tsujimoto Y. Acinus is a caspase-3 activated protein required for apoptotic chromatin condensation. Nature 1999; 401: 168-173.

13. Rappsilber J, Ryder U, Lamond AI, Mann M. Large-scale proteomic analysis of the human spliceosome. Genome Res 2002; 12: 1231-1245.

14. Schwerk C, Prasad J, Degenhardt K, Erdjument-Bromage H, White E, Tempst $P$ et al. ASAP, a novel protein complex involved in RNA processing and apoptosis. Mol Cell Biol 2003; 23: 2981-2990.

15. Joselin AP, Schulze-Osthoff K, Schwerk C. Loss of Acinus inhibits oligonucleosomal DNA fragmentation but not chromatin condensation during apoptosis. J Biol Chem 2006; 281 12475-12484.

16. Tange TO, Shibuya T, Jurica MS, Moore MJ. Biochemical analysis of the EJC reveals two new factors and a stable tetrameric protein core. RNA 2005; 11: 1869-1883.

17. Hu Y, Yao J, Liu Z, Liu X, Fu H, Ye K. Akt phosphorylates acinus and inhibits its proteolytic cleavage, preventing chromatin condensation. EMBO J 2005; 24: 3543-3554.

18. Kato T, Muraski J, Chen Y, Tsujita Y, Wall J, Glembotski CC et al. Atrial natriuretic peptide promotes cardiomyocyte survival by cGMP-dependent nuclear accumulation of zyxin and Akt. J Clin Invest 2005; 115: 2716-2730.
19. Bavelloni A, Faenza I, Cioffi G, Piazzi M, Parisi D, Matic I et al. Proteomic-based analysis of nuclear signaling: PLC $\beta 1$ affects the expression of the splicing factor SRp20 in Friend erythroleukemia cells. Proteomics 2006; 6: 5725-5734.

20. Borgatti P, Martelli AM, Tabellini G, Bellacosa A, Capitani S, Neri LM. Threonine 308 phosphorylated form of Akt translocates to the nucleus of $\mathrm{PC} 12$ cells under nerve growth factor stimulation and associates with the nuclear matrix protein nucleolin. $J$ Cell Physiol 2003; 196: 79-88.

21. Xuan Nguyen TL, Choi JW, Lee SB, Ye K, Woo SD, Lee KH et al. Akt phosphorylation is essential for nuclear translocation and retention in NGF-stimulated PC12 cells. Biochem Biophys Res Commun 2006; 349: 789-798.

22. Fu H, Subramanian RR, Masters SC. 14-3-3 proteins: structure, function, and regulation. Annu Rev Pharmacol Toxicol 2000; 40: 617-647.

23. Brunet A, Kanai F, Stehn J, Xu J, Sarbassova D, Frangioni JV et al. 14-3-3 transits to the nucleus and participates in dynamic nucleocytoplasmic transport. J Cell Biol 2002; 156: 817-828.

24. Liu X, Kim CN, Yang J, Jemmerson R, Wang X. Induction of apoptotic program in cell-free extracts: requirement for dATP and cytochrome c. Cell 1996; 86: 147-157.

25. Nix DA, Fradelizi J, Bockholt S, Menichi B, Louvard D, Friederich E et al. Targeting of zyxin to sites of actin membrane interaction and to the nucleus. J Biol Chem 2001; 276: 34759-34767.

26. Faul C, Huttelmaier S, Oh J, Hachet V, Singer RH, Mundel P. Promotion of importin alphamediated nuclear import by the phosphorylation-dependent binding of cargo protein to 14 3-3. J Cell Biol 2005; 169: 415-424.

27. Ahn JY, Rong R, Kroll TG, Van Meir EG, Snyder SH, Ye K. PIKE (phosphatidylinositol 3kinase enhancer)-A GTPase stimulates Akt activity and mediates cellular invasion. J Biol Chem 2004; 279: 16441-16451.

28. Ye K, Compton DA, Lai MM, Walensky LD, Snyder SH. Protein $4.1 \mathrm{~N}$ binding to nuclear mitotic apparatus protein in PC12 cells mediates the antiproliferative actions of nerve growth factor. J Neurosci 1999; 19: 10747-10756.

29. Ye K, Ke Y, Keshava N, Shanks J, Kapp JA, Tekmal RR, Petros J, Joshi HC. Opium alkaloid noscapine is an antitumor agent that arrests metaphase and induces apoptosis in dividing cells. Proc Natl Acad Sci USA 1998; 95: 1601-1606. 